\title{
Structural Bus and Release Mechanisms on the ST5 Satellites -Summary and Status ${ }^{12}$
}

\author{
Peter Rossoni \\ NASA Goddard Space Flight Center \\ Mail Code 544 \\ Greenbelt, MD 20771 \\ 301-286-5854 \\ Peter.Rossoni@gsfc.nasa.gov
}

Abstract - The Space Technology 5 Mechanical System met the challenge of packaging a fully functional science and technology satellite system with its Deployer mechanism into a compact $0.07 \mathrm{~m}^{3}$ volume. Three $25 \mathrm{~kg}$ satellites were orbited in constellation in March, 2006.

The ST5 mechanical system is composed of

- The Structural Bus

- Magnetometer Instrument Boom

- Spacecraft Deployer Release Mechanism

This system includes a highly integrated electronics enclosure as a multifunctional structure; a lightweight, magnetically clean Magnetometer Boom; the first use of Nitinol Shape-Memory Alloy trigger devices for deploying multiple spacecraft; an innovative compliant mount for the umbilical connector and a Deployer mechanism that imparts both separation velocity and mission spin rate to three constellation flying satellites

These elements employed cutting-edge design and analysis tools, state-of-the-art testing facilities and proven engineering techniques to meet stringent performance criteria, enabling the mission's success.

\section{TABLE OF CONTENTS}

1. INTRODUCTION 1

2. STRUCTURAL BUS .......................................................3

3. MAGNETOMETER BOOM ...........................................9

4. DEPLOYER STRUCTURE ........................................... 10

5. ON-ORBIT PERFORMANCE ....................................... 13

6. CONCLUSION .................................................................. 13

REFERENCES..................................................................... 14

BIOGRAPHY .................................................................... 14

\section{INTRODUCTION}

Mission and Spacecraft Overview -The Space Technology 5 mission was a 3-satellite constellation demonstrator for miniaturized spacecraft technology. At the same time, it acquired high quality science data in the Earth's magnetosphere, making new discoveries over the course of its mission. Figure 1 shows an artist's conception of the constellation over the Arctic with the Aurora Borealis (a magnetic phenomenon) in the background.

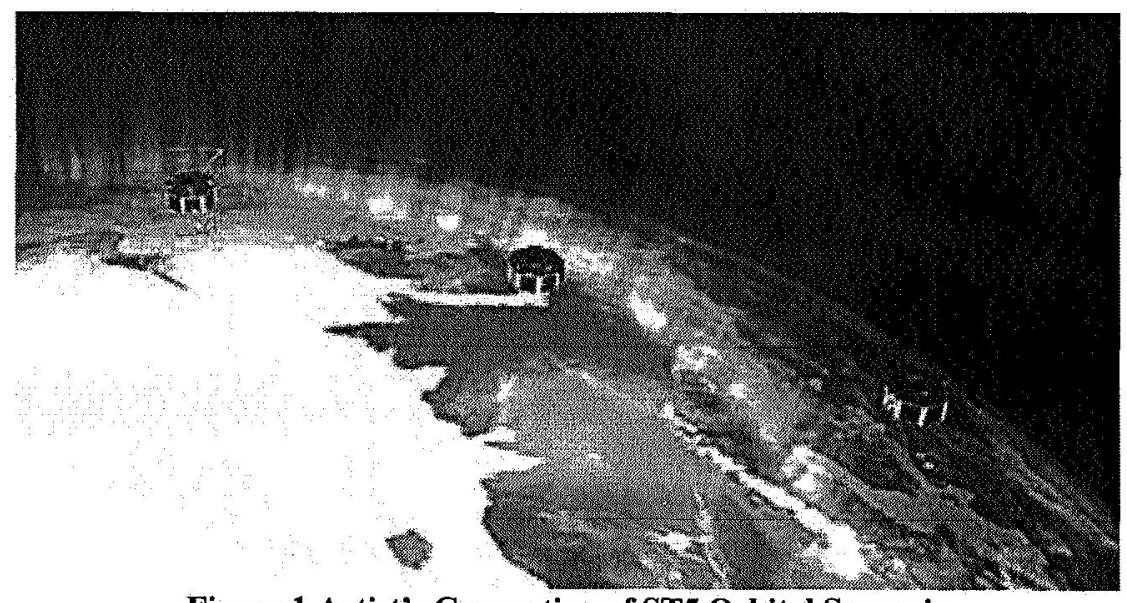

Figure 1 Artist's Conception of ST5 Orbital Scenario

\footnotetext{
1 IEEEAC paper \#1307, Version 0

${ }^{2}$ U.S. Government work not protected by U.S. copyright
} 
The $25 \mathrm{~kg}$, 20 watt spacecraft constellation carried the following technologies:

- X-Band transponder for both up- and down-link communications and ranging

- Miniaturized thruster with cold gas tank

- Lithium-Ion battery

- Variable Emittance Thermal Control technologies for active temperature control

- Ultra-Low Power, Radiation Tolerant logic circuit

- Ground Operations methods for constellations

The technologies were validated during its mission from March $22^{\text {nd }}$ to June $30^{\text {th }}, 2006$. The ST5 success paves the way for future missions utilizing constellations of smaller, more capable spacecraft.

\section{Mechanical System Requirements [1,2]}

Volume -The mechanical system was impacted by volume constraints because it was originally planned for launch as a secondary payload. The design was constrained to a $60 \mathrm{~cm}$ cubic envelope $\left(0.22 \mathrm{~m}^{3}\right)$ per spacecraft, including extensions for deployable appendages. The 30 layer C\&DH board as well was quite small with respect to comparable systems on other spacecraft. Nevertheless the C\&DH made up the entire height and width of the ST5 structural bus. Twice during conceptual design the bus was enlarged to accommodate growth in the C\&DH.
Each spacecraft could be placed separately according to available accommodations on a large Evolved Expendable Launch Vehicle (EELV). When a smaller, primary launch opportunity was found on the Pegasus Launch Vehicle, the compact, modular design was reconfigured onto a support structure as shown in Figure 2. This packaged all three satellites into its $120 \mathrm{~cm}$ diameter, $180 \mathrm{~cm}$ long payload envelope.

Magnetic Cleanliness -Due to the requirement for highquality science data retrieval, all materials used on the spacecraft and Pegasus Support structure underwent extensive magnetic signature review and mitigation. All components and subsystems were required to keep their signature below 10 nanoTesla (nT) DC and 50 nT AC. The closer a component was to the magnetometer, the more scrutiny it received. Actively induced Magnetic fields such as from electrical power generation in the solar panels were minimized as well.

The magnetometer boom, since it carried the instrument itself, received some of the most stringent scrutiny for magnetic cleanliness. All exposed metal surfaces were coated to reduce temperature gradients and excursions. This reduced the thermo-magnetic effect. Even the standard low-magnetism stainless steel fasteners were replaced with custom made Titanium screws.

Other requirements -The boom's natural mode of vibration was kept within the dead-band of the science instrument to prevent possible contamination of data; the magnetometer and its harness were wrapped in blanketing to minimize heat loss.

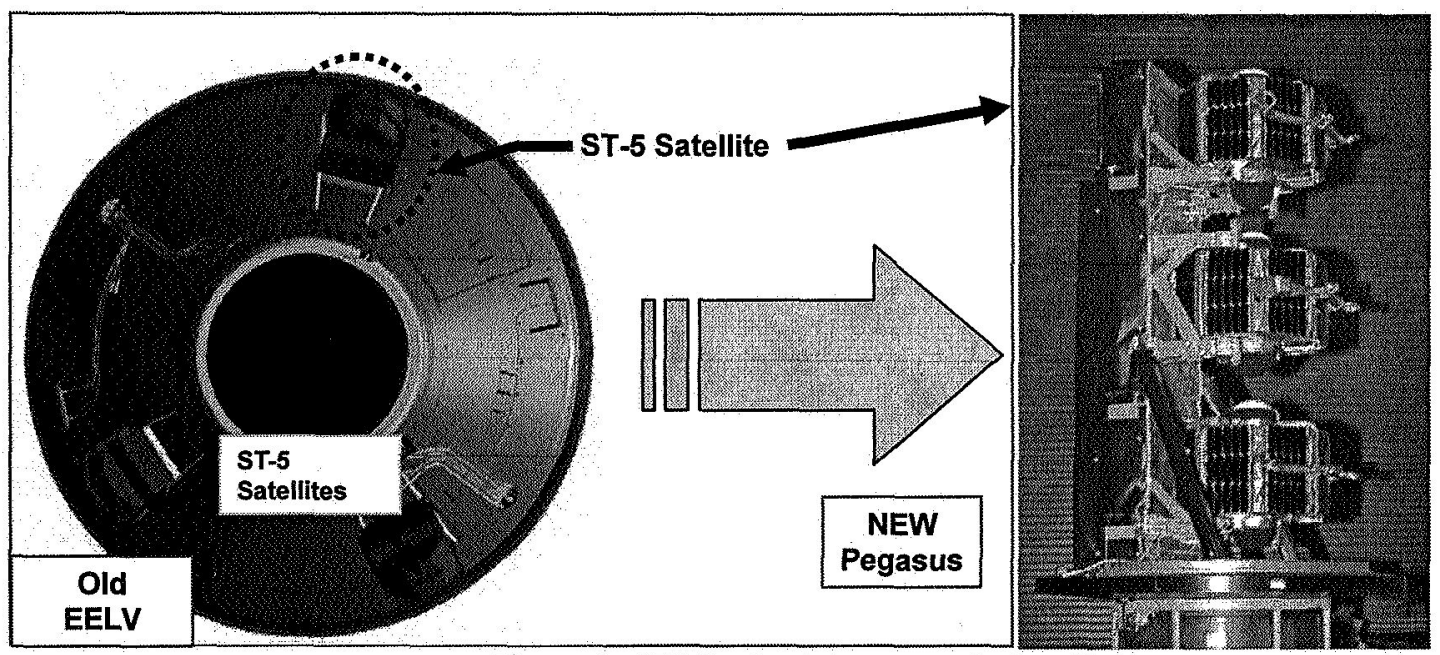

Figure 2 ST5 Payload Accommodation Change

-Discrete Locations on EELV Payload Attach Fitting to Single Co-planar Mounting Surface on Pegasus

The spacecraft was stabilized with a spin rate of 20 RPM

$\pm 10 \%$ initially to assure good data retrieval and proper 
functioning of the attitude determination and control components. In addition, the spacecraft layout was carefully balanced to minimize "wobble." The requirement was to orient the spin axis (Principal Moment of Inertia) with the geometric $Z$ axis to within 1 degree. A derived requirement from spin stabilization stipulated the Principal moment of inertia in the spin axis had to be at least $10 \%$ greater than any other inertia.

The release mechanism was tested thoroughly for reliability and to minimize the chance of re-contact between departing spacecraft and launch vehicle. A doubly redundant set of limit switches on the spacecraft bus top deck turned on power to each spacecraft as it deployed. At the same time, a "flyaway" connector indicated separation status to both the spacecraft and the launch vehicle.

System Description -Each spacecraft bus is an octagonal aluminum structure composed of top and bottom decks and held together by a cast aluminum backbone. The backboneto-deck joint provides a rigid attachment point to the release mechanism. Each vertical facet of the octagon supports a solar panel of honeycomb sandwich construction with graphite face sheets.

The booms may be seen in Figure 2 wrapped around three facets of the spacecraft octagonal sidewall. The boom is composed of three graphite composite tube segments connected by "carpenter tape" hinges. A mechanism at the tip (near the magnetometer seen on the middle spacecraft) releases the boom, which then unfolds and snaps open. The boom attachment to the spacecraft body is seen on the top and bottom spacecraft.
The deployer release mechanism is composed of three struts or stanchions with mechanisms at the top which hold the spacecraft attach points. The top and bottom spacecraft were mounted "upside down" as shown in the picture to place their bottom decks with umbilical connectors within reach of available launch vehicle fairing access doors.

This paper will discuss significant design and validation efforts and how major issues and concerns were addressed in the course of the mechanical system development and delivery to orbit.

\section{StRUCTURAL BUS}

The main parts of the Structural Bus were:

- Aluminum top and bottom decks

- A "Card Cage" structural backbone that tied the decks together

- Sidewalls

These are shown in figure 3.

Decks - The machined aluminum decks were originally designed as honeycomb sandwich construction. Since the spacecraft was so small compared with the internal components it supported, the additional performance from the honeycomb was found to be a small benefit compared with the extra work involved in designing and qualifying it. In addition, the electrical and thermal properties of the single-material aluminum deck were much desired by the power and thermal engineers. As in may other applications, the decks served as heat sinks and radiators for thermal control as well as chassis ground for the electrical system.

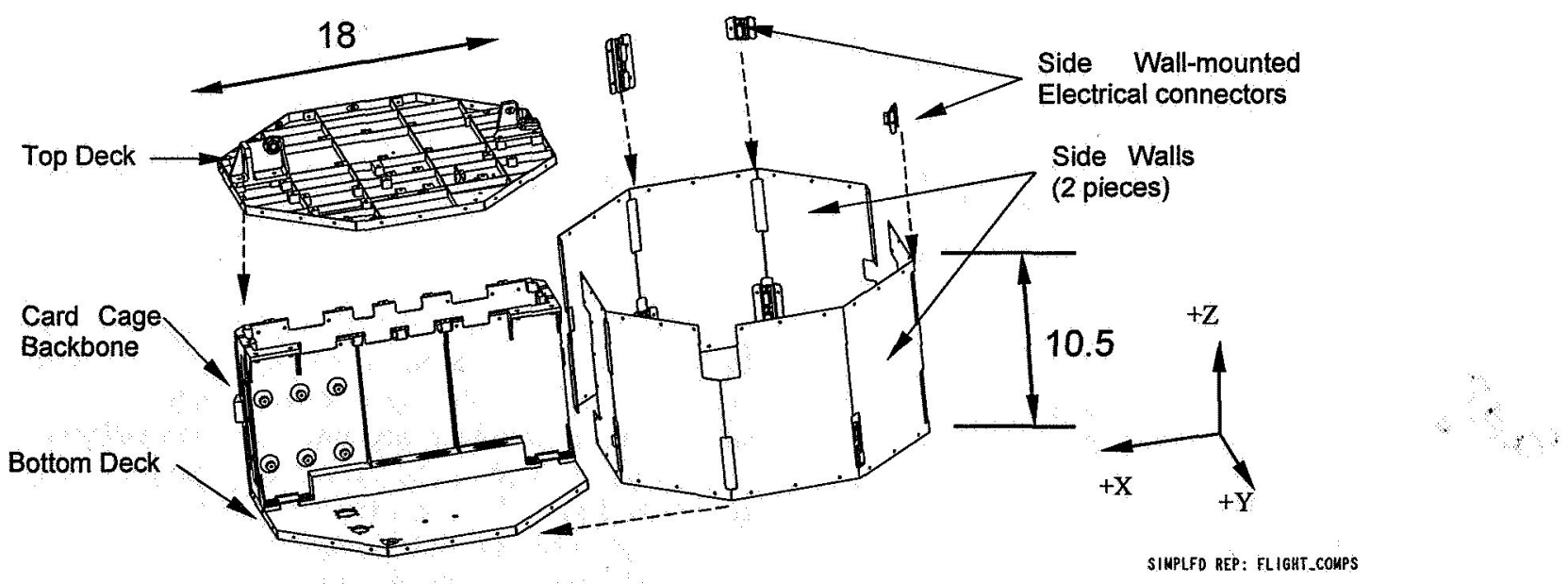

Figure 3 ST5 Structural Bus Elements

Mechanical Packaging -

After settling on placement and contents of the card cage, the remaining area was available for the rest of the components. Most desirable was to place components 
on either of the two decks, for structural rigidity as well and for heat dissipation. Unfortunately, volume and area in the decks ran out fairly quickly for the high-heat generating components as well as those which required placement there for other reasons. The sheet metal exterior sidewall of the spacecraft supported not only exterior connectors and solar panels, but also a current limiter for the Transponder, boom snubbers and the Nutation Damper. The Card Cage exterior served to support two electronics units for the thermal control experiments as well as the Thruster Control Electronics. Cast-in bosses were provided in the card cage wall for mounting these three boxes, which weighed approximately 370 grams for the TCE and 220 grams each for the VEC boxes. In addition, the majority of harnessing going to and from the CCA was supported by the CCA walls. Aluminum tie-wrap clips were bonded in strategic locations to secure the harness with conductive epoxy in order to eliminate all possible floating grounds.

In order to verify that the extra loading on the Card Cage wall would not affect the internal electronics, the Finite Element Model (FEM) as shown in Figure 4 included both rigid masses representing the combined $800 \mathrm{~g}$ weight of these elements plus the extra "smeared mass" for the harnessing. The resultant stresses were well within acceptable limits.

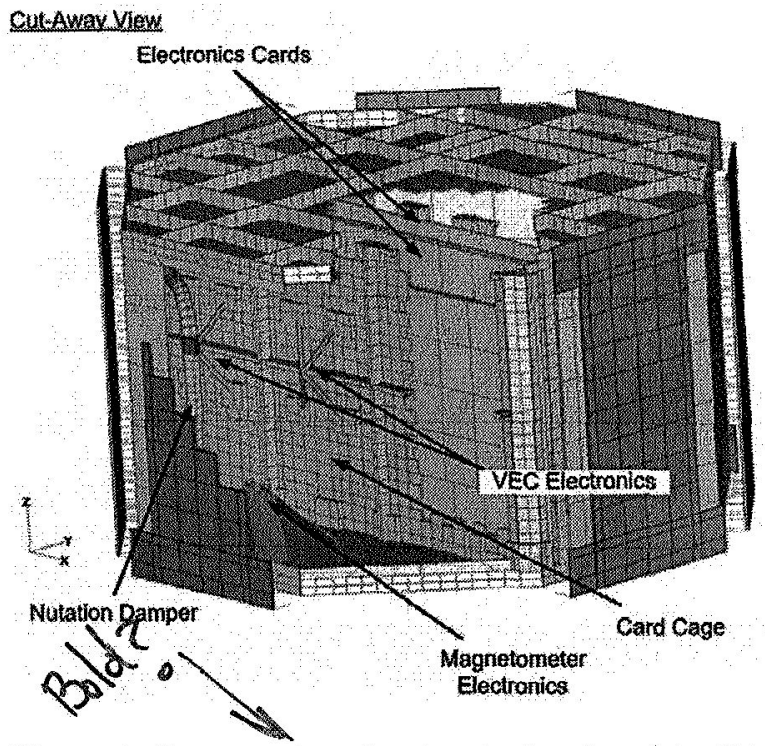

Figure 4: Cutaway view showing the interior of the ST5 bus structure FEM

"Card Cage" Electronics Enclosure - Key to keeping the spacecraft volume low was making the best use of all available space. To this end, the structural backbone was made hollow into a "Card Cage" to accommodate the Command and Data Handling (C\&DH) computer and the Power Systems Electronics (PSE) cards. This investment-cast part was lighter than the electronics it held. Not only that, all structural loads bypassed the boards, going through the walls and deck attach screws instead.

Card Cage Fabrication - The investment casting technique involves several stages. First, a temporary pattern was created representing the exact dimensions of the card cage. Features to support the part and enable proper flow of the molten metal were added. Second, the pattern was repeatedly immersed in ceramic slurry, coating and drying it between layers resulting in a mold. The mold was fired, burning out the temporary pattern and leaving a cavity for the finished product. Upon cooling and touch-up, the mold was ready for pouring. Molten casting aluminum, grade A357, was manually poured into the mold and allowed to cool. Finally, the mold was broken, gates and sprues ground off, and the part was grit-blasted, heat treated and finish machined to its final dimensions.

Though at first glance this technique appears to be labor and time intensive, in fact for multiple parts, made in parallel fashion, it is very efficient. Complex features are easily integrated, and the patterns (and resulting parts) can hold tight dimensional tolerances. The as-cast electronics slots, for example, were held to 0.005 " tolerance to keep boards straight as well as $32 \mathrm{rms}$ finish for high heat transfer.

Features of the card cage are shown in Figure 5. A backplane Printed Wiring Board joined the PSE and C\&DH electronics. The boards were inserted from the card cage top into the vertical slots. Interface connectors on the board bottom edge mated with the backplane, and connectors to outside the card cage slid into their accommodations on the card cage wall top edge. The backplane also had external connectors. In all, the card cage had eleven external connectors and four internal ones.

The card cage was qualified for flight by analysis. At first, two quick-turn-around, prototype models were created from Rapid-Prototype mode patterns. These were not as dimensionally accurate as the wax pattern to be used on the flight units, but served to prove out the concept and get solid, early results from environmental testing and board fit- and functional checks. One of these card cages went through vibration test along with the rest of the structural bus, since they are so closely integrated.

Since the first units' dimensions such as wall thickness were not optimized for flight, it was not a true 
qualification unit. Standard procedures at GSFC allow for qualification by analysis as long as the factor of safety used in calculating stress margins is increased from 1.25 to 2.0 . Confidence in the design led to this approach. In the end, the card cage was officially qualified by analysis, but in fact also proved out during environmental test as an integrated part of the bus.

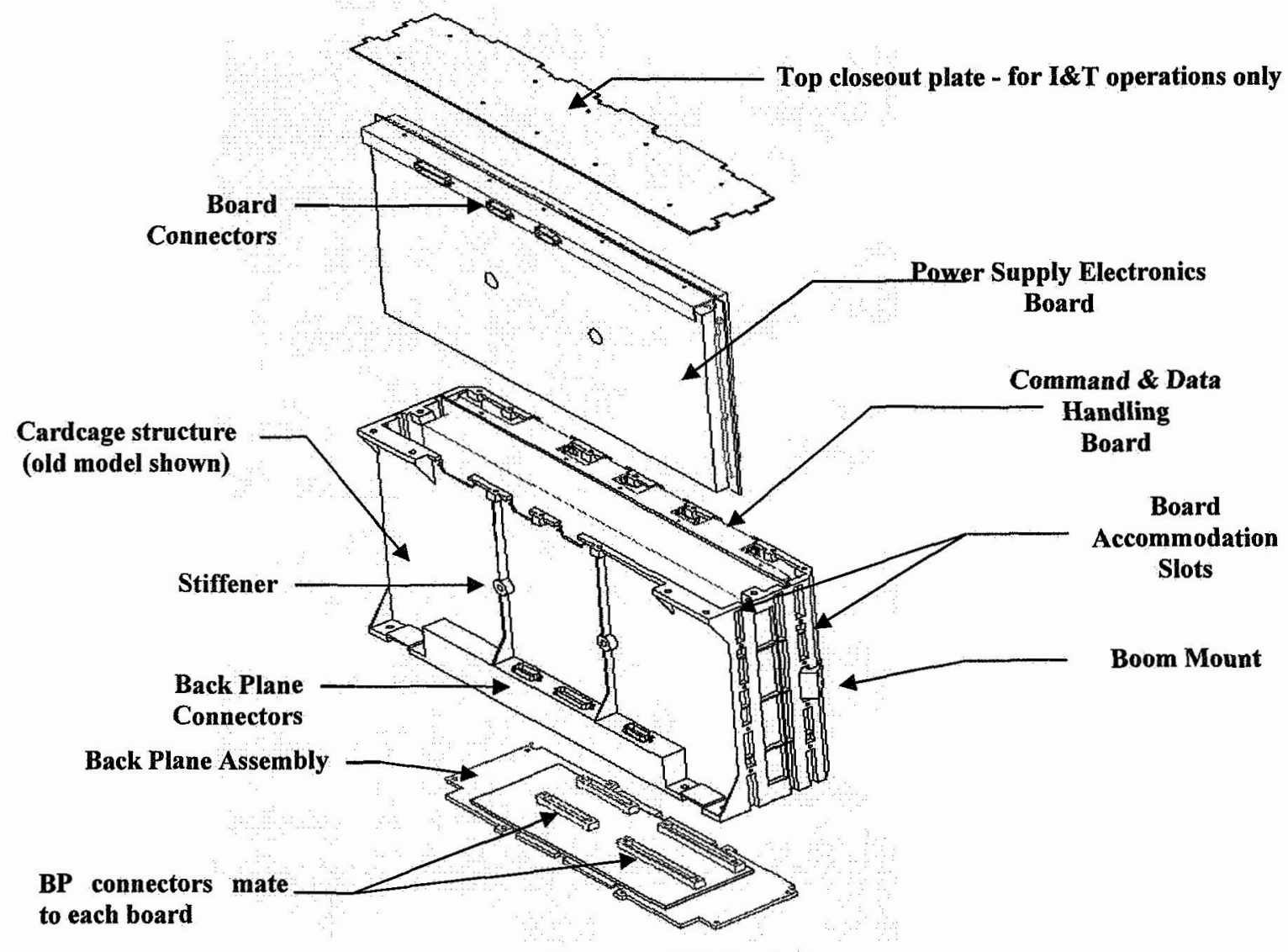

Figure 5 ST5 Card Cage

Bus Mass Properties - The ST5 spacecraft mass properties testing consisted of mass, Center of Gravity (CG), Moments of Inertia (MOI) and Products of Inertia (POI). All these measurements were key to determining the on-orbit trajectory and spin characteristics of the spacecraft. The most stringent requirement was to assure that the spacecraft spins true along its vertical $\mathrm{Z}$ axis (Figure 3), with a minimum of wobble once separated from the launch vehicle. As the design progressed, the components were positioned on the decks in order to optimize the assembly balance both statically and dynamically. The design process counted major mass items, however even distribution of masses was hampered by the constrained space available and it did not take into account the significant mass effect of electrical harnessing and thermal control blankets. Harnessing and blankets contributed over $10 \%$ of the spacecraft mass. Provision was made in the mass budget for balance weights up to $1 \mathrm{~kg}$ per spacecraft to compensate for these and other unknowns in the final SC mass properties.

Spin Balance Testing - The process for spin balancing involves measuring the torque caused by the spacecraft's wobble. This torque, also known as Products of Inertia (POI) causes the spacecraft's spin axis in free space to shift away from the desired $(Z)$ axis in what is called 
coning. This torque increases as the square of the spin rate about a given axis, so it is necessary to spin test at the maximum rate practically achievable.

Fortunately the ST5 spacecraft test did not include the boom, which would have encountered significant air resistance at that rate. The boom of course needed to be factored in to the final balance results. We investigated running the test with the boom attached; it could have been conducted in a vacuum chamber or by mounting an air shield with vanes around the spacecraft in order to move the air with the boom. Goddard has two thermal vacuum chambers large enough to hold the test facility, which is vacuum-rated down to 10 torr $(1.3 \mathrm{kPa})$. The vacuum chamber option was eliminated because the cost associated with certifying, designing and carrying out the test would have been high. The air shield idea made it to the prototype stage when it was discovered that the bulk of the sheet metal shield required tremendous mass to maintain stiffness for adequate balance (the support hardware for a spin balance test must be much more repeatable than that of the flight hardware). In the end, it was simpler to test without the boom, then for flight, mount the boom's center of gravity on the same plane as the SC center of gravity. This was accomplished with adapter plates for each of the four boom attach points to the spacecraft.

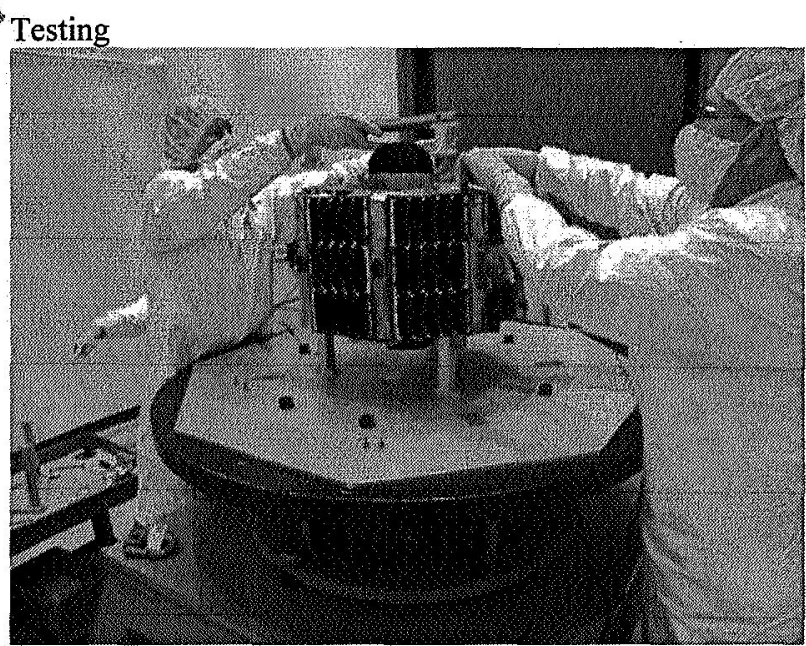

Figure 6 ST5 on the MPMF at Goddard

The challenges in performing the balance included the relatively small SC size and the large capacity of the test facility. Figure 5 shows the test setup on Goddard's Mass Properties Measurement Facility (MPMF). This facility measures the torque as the spacecraft spins about the desired balance axis on a hemispherical air bearing. The MPMF SC testing capacity is up to 10,000 pounds, so reaching the desired accuracy for the $55 \mathrm{lb}$ ST5 spacecraft was a challenge. To compensate, the spin rate exceeded 130 RPM to adequately assess the unbalance and correct it. Also the weights had to be placed on an already busy and space-constrained deck.

Measurements of the unbalance are made with the internal force sensor and digital smoothing techniques. Figure 6 shows some sample raw data acquired with \pm 2 in-lb resolution. Since sampling occurred at $5 \mathrm{kHz}$, at 130 RPM 2500 samples were taken during one spin period. Circular curve fitting yields a precise torque value for a given spin rate.

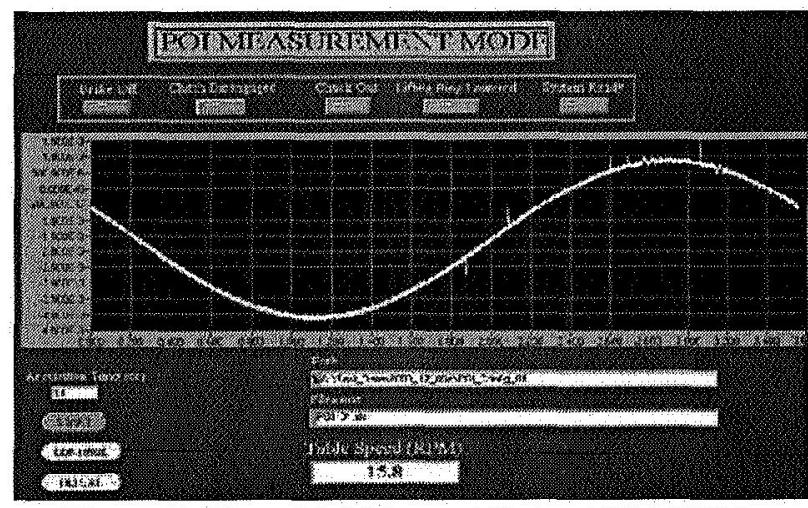

Figure 7 Sinusoidal Moment Data at 15.8 RPM

Data sets of moment versus spin rate squared for two iterations are shown in Figures $7 \mathrm{a}$ and $7 \mathrm{~b}$. The slope of the best-fit straight line through the data yields the spacecraft unbalance. The goal of the test is to minimize this slope (zero slope would indicate zero unbalance). The process is completed by calculating, designing and adding balance weights to the decks' outer perimeter.

Spin Balance Sequence -When first integrated, the spacecraft underwent a coarse mass properties and spin balance measurements early in the test flow. Preliminary weights were attached, and the CG, MOI and POI were re-measured to complete the first iteration. At the end of the test flow, before shipping to the launch site, a fine spin balance test was conducted and the weights were refined. In the end the weights weighed less than $1 \mathrm{~kg}$ per spacecraft and their positions on each SC corresponded to within $5 \mathrm{~cm}$. This showed that the three SC were manufactured consistently, despite having a fair amount of manual construction involved. 

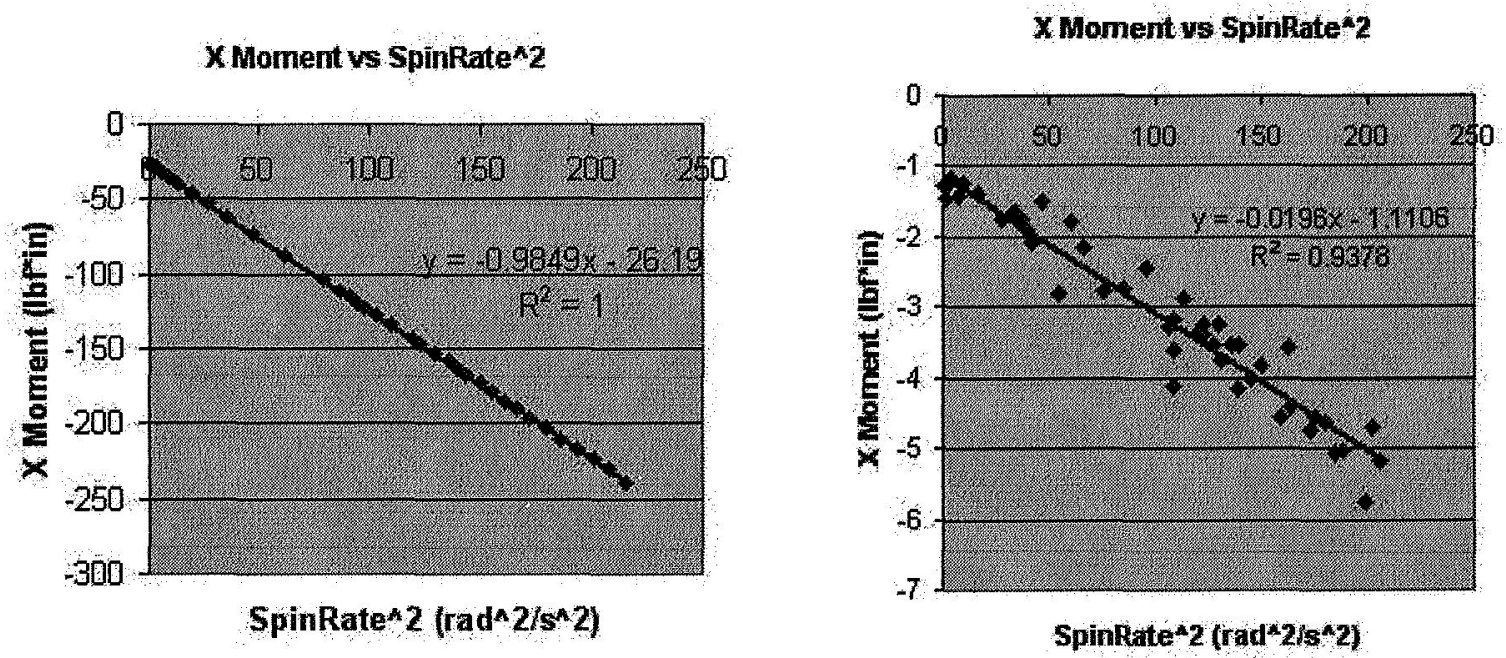

Figure 7 a \& b. Moment Data Results -First and Second Iterations

Solar Panels

Each ST5 spacecraft carried eight solar panels populated with $28 \%$ efficient photovoltaic cells. These were research stage cells, not available commercially at the time. Each panel had three strings of 5 cells. Characteristics are shown in Table 1. Current generated in each string of cells was passed back directly under the string to cancel the magnetic field. This technique reduced what would ordinarily be a 50 nano-Tesla to a field on the order of 5 pico-Tesla. The panel substrate seen in Figure 8 carried twice its own mass in power equipment, and was fabricated with a Kapton insulating layer co-cured in the top facesheet (seen brown along the edges).
Table 1 ST5 Solar Array Characteristics

\begin{tabular}{|l|l|}
\hline Power & $23 \mathrm{~W} @ 9.2 \mathrm{~V}$ \\
\hline Panel Total Area & $73 \mathrm{in}^{2}$ \\
\hline Active area & $60 \mathrm{in}^{2}(81 \%$ usage $)$ \\
\hline Cover Glass & 0.020 "/ITO Coated \\
\hline Electrical mass & $180 \mathrm{grams}$ \\
\hline Panel substrate mass & 80 grams \\
\hline Magnetic Cancellation & $\begin{array}{l}\text { Silver } \\
\text { Underlayment }\end{array}$ \\
\hline
\end{tabular}

Panel Fabrication - The panel's honeycomb construction created a light and stiff substrate for the cells. Four plies of 0.0025 " thick graphite composite made up each facesheet for a thickness of only 0.010 ". Overall, the panel dimensions were 11.25 " long by 6.5 " wide by $0.155^{\prime \prime}$ thick, not including cover glass, cells or silver mesh field cancellation.

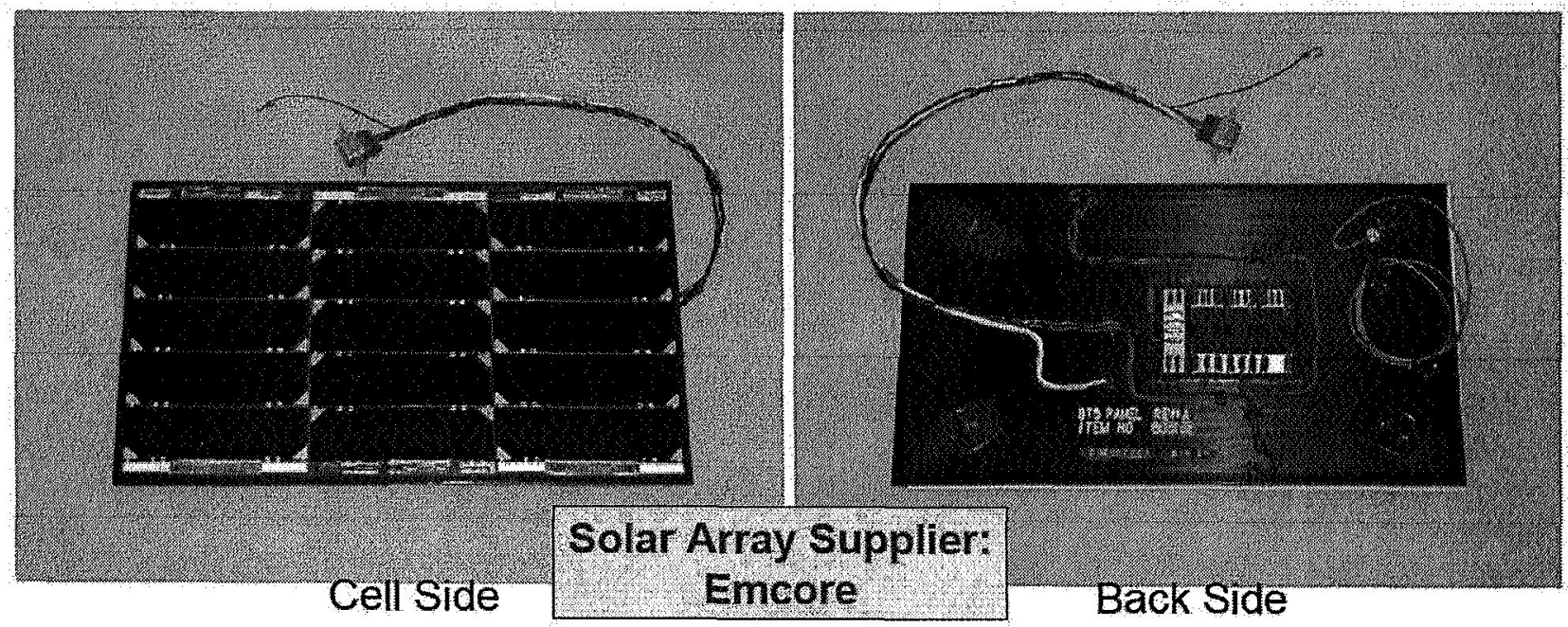

Figure 8 ST5 Solar Panel 
The panels were fabricated in a bulk sheet large enough to produce eight panels. Flatness of the tooling plate used during cure determined the flatness of the finished product. Before cutting out the individual panels, the sheet was subjected to Ultrasonic inspection shown in Figure 8. Any potential flaws were highlighted and used for test coupons or avoided entirely for the final product. Small squares served as flatwise tension coupons (testing the adhesion of the honeycomb to the facesheet); the small rectangles served as flexure coupons (testing the stiffness of the construction) and the large rectangles were the flight panels. The coupons and panels were machined to final dimensions on a precision CNC routing machine, which also placed precision alignment holes for installing the panel's mounting flexures.

The qualification panel ultrasound image after all testing is shown in Figure 9. No flaws are seen. The four flexures and identification tag show up clearly.

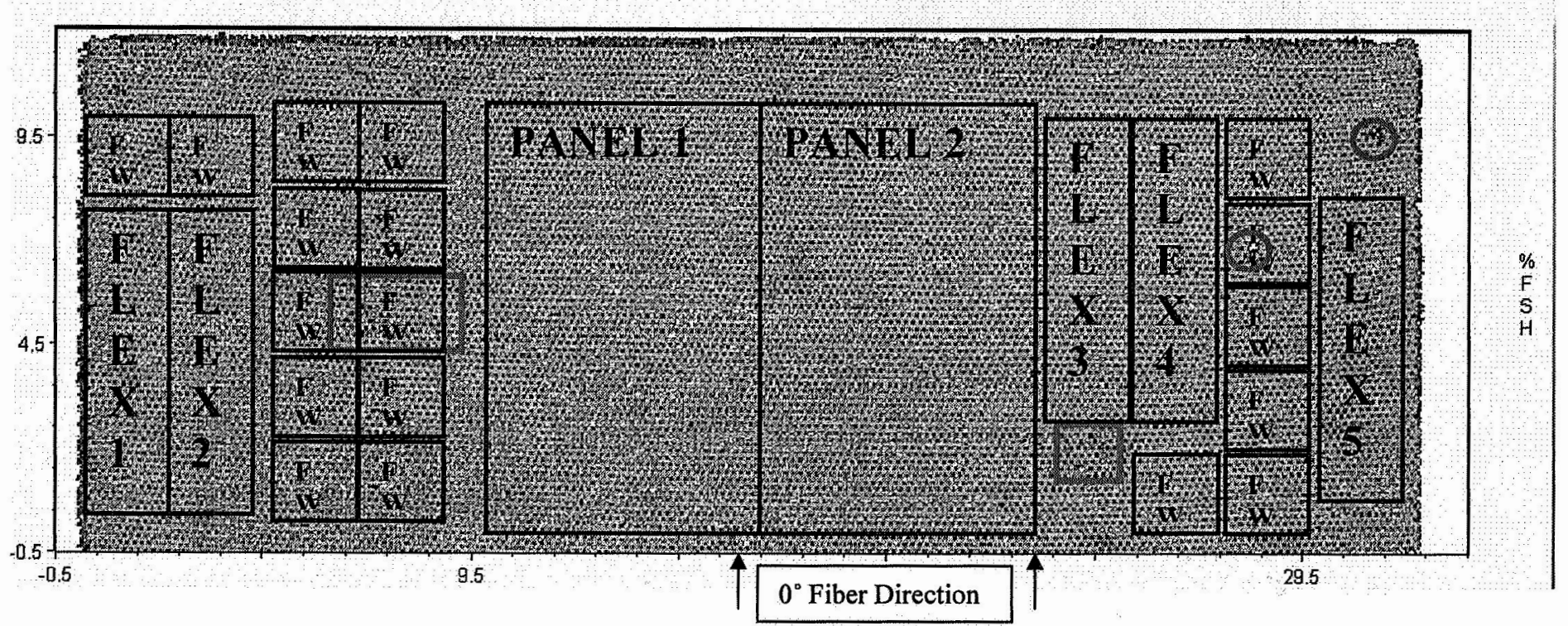

Figure 9 ST5 Solar Panel Honeycomb Sheet Ultrasound Image

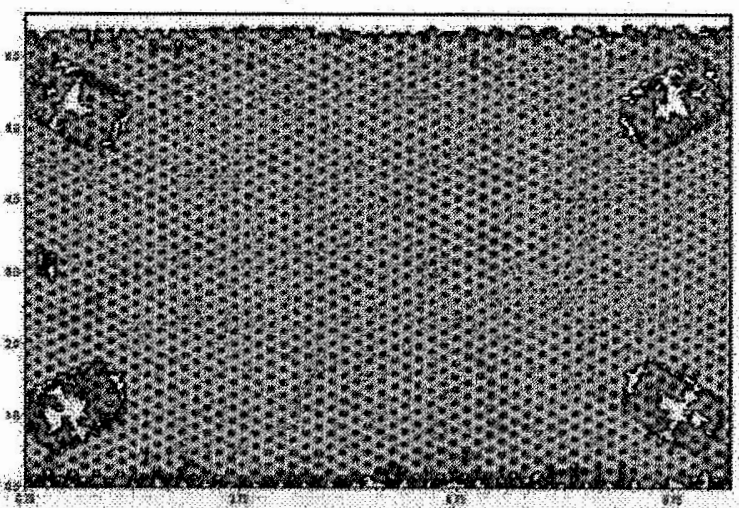

Figure 10 ST5 Post-Qualification Solar Panel Ultrasound Image 


\section{MAGNETOMETER BOOM}

The instrument boom shown in Figure 10 carried a 3-axis research-quality flux-gate magnetometer. In order to keep the science data as free as possible from contamination from the spacecraft itselif, the boom deployed on-orbit to place the instrument at least $1.5 \mathrm{SC}$ diameters from the closest point on the structure. $\bigcirc \mathrm{SC}$ ?

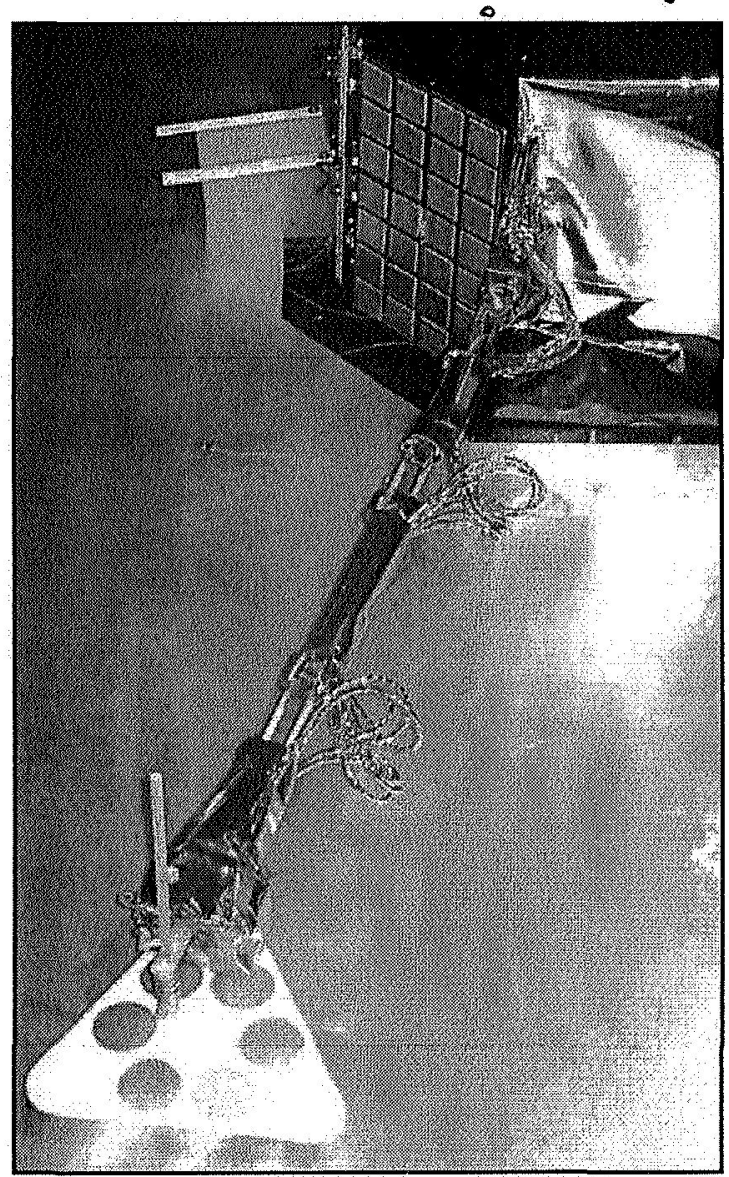

Figure 11 ST5 Magnetometer Boom in Qualification Thermal Test

The hinges connecting each segment were composed of opposing pairs of Beryllium-Copper sheet strips similar to carpenter tape. When bent, the hinges collapsed as shown in Figure 11.

The three folds allow the boom to stow around the spacecraft solar panels. "V" shaped snubbers keep the boom off the solar cells, and provide tension to hold the boom in place during launch.

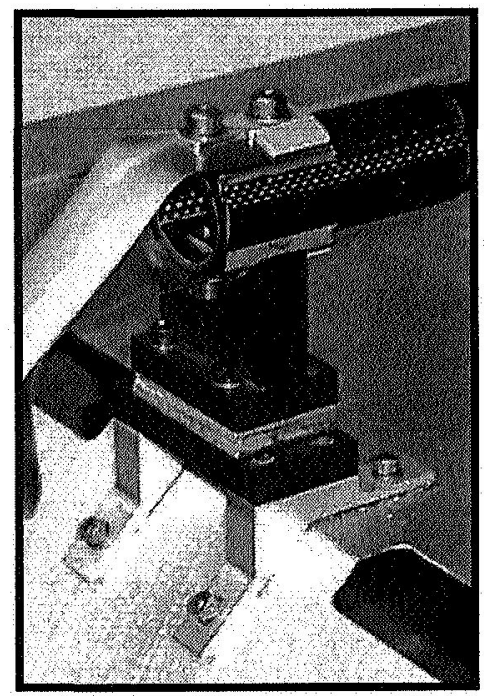

\section{Figure 12 ST5 Magnetometer Boom Hinge Stowed}

When deployed on orbit, the internal strain energy and centrifugal acceleration cause the boom to extend and snap in its final straight, rigid shape.

Figure 10 shows the triangular G-Negation Trolley at the boom tip, which allowed the boom to deploy smoothly even under gravity.

\section{Boom Requirements}

Table 2 Magnetometer Boom requirements

\begin{tabular}{|l|l|}
\hline Magnetic Signature & $<0.5 \mathrm{nT}$ \\
\hline Stability & $1 / 4^{\circ} ; 1 \mathrm{~cm}$ (when deployed) \\
\hline Natural Frequency & $5-11 \mathrm{~Hz}$ (rigid base) \\
\hline Amplitude & $\begin{array}{l}95 \% \text { within 20 seconds of } \\
\text { deploy }\end{array}$ \\
\hline
\end{tabular}

\section{Magnetic Cleanliness}

Since the boom was in intimate contact with the science instrument, it has been designed to minimize magnetic interference with the magnetometer and provide thermal and dynamic stability. All exposed metal was coated in order to reduce temperature gradients, since magnetic fields are generated in a conductor due to the thermo-magnetic effect. The standard, low-magnetism A286 stainless steel fasteners used on the rest of the spacecraft were replaced with even "cleaner" brass screws to attach the instrument, and Titanium screws for the hinges. The hinges have a gold coating 50 microns thick.

\section{Ground Test}

The magnetometer boom was subjected to full qualification testing separate from the spacecraft, including vibration, alignment and thermal testing at flight levels. The boom 
was in its flight configuration; i.e., flight-like harnessing and blankets installed.

The natural frequency was demonstrated to be $>10 \mathrm{~Hz}$. Damping was sufficient to reduce peak-to-peak displacement by $95 \%$ in $\sim 15$ seconds.

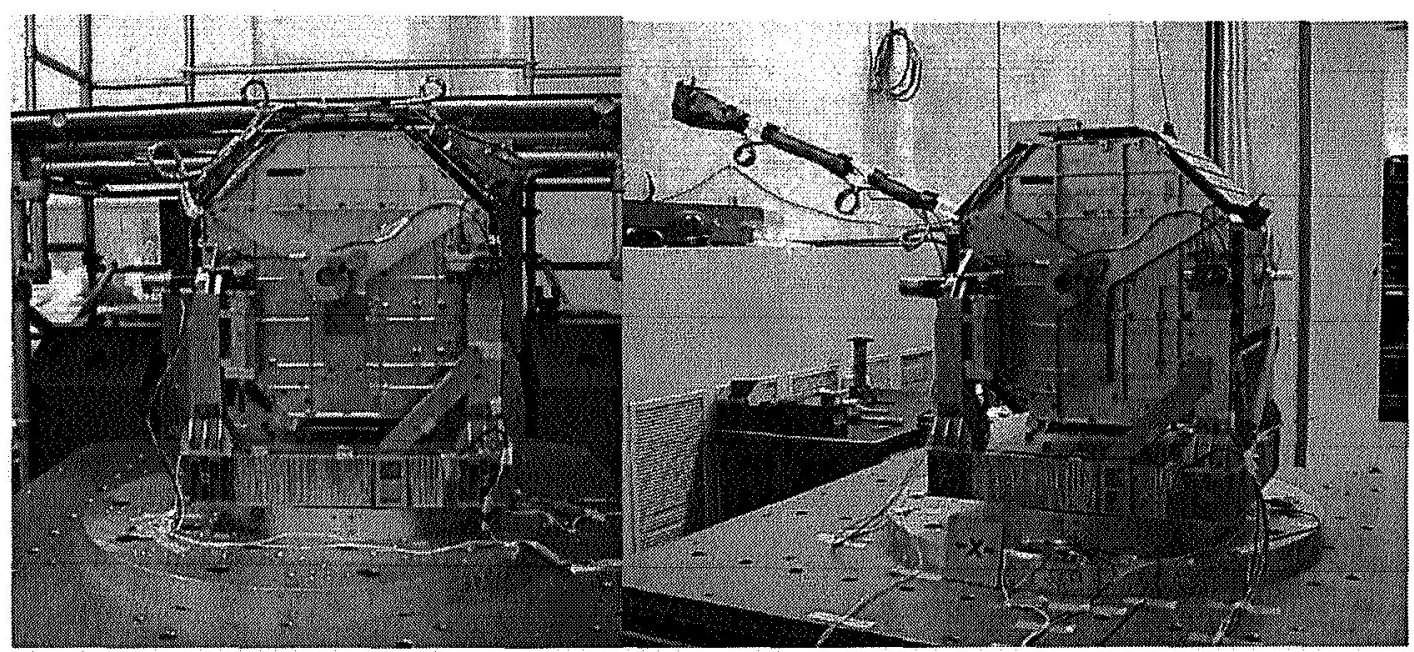

Figure 13 ST5Magnetometer boom, stowed (left) and deployed (right)

\section{Challenges}

- Diameter and resonance requirements

- Wrap-around design and underpowered pinpuller

- Hinge cracking Structural loads

\section{Deployer StruCture}

\section{Description}

The Spacecraft release mechanism, called the Deployer Structure, releases each spacecraft from the launch vehicle while imparting a nominal spin. The mechanism supports the entire spacecraft, and provides the electrical interface to the launch vehicle, including providing separation indication to both the spacecraft and back to the ground. ST5 demonstrated the first use of a low-shock shapememory alloy pinpuller device to initiate spacecraft deployment. The spacecraft separation connector, on a compliant mount, featured a high tolerance for misalignment, while allowing for separation with zero force. The Deployer Structure design is modular, and could be used as-is for future missions. As described earlier, three separate Deployer Structures were incorporated onto a newly designed Pegasus Support Structure.

Operation of the Deployer Structure is described in Figure 13. The spacecraft is first kicked off on the left hand side when the Pinpuller trigger retracts and the pusher starts clockwise rotation. The SC pivots about hinge pins on the right side until the pusher has completed its stroke. Then the hinges, which are mechanically timed, retract at about 20 degrees of rotation. The $\mathrm{SC}$ is then free to translate away from the launch vehicle at a rate of $1 / 2$ meter per second.

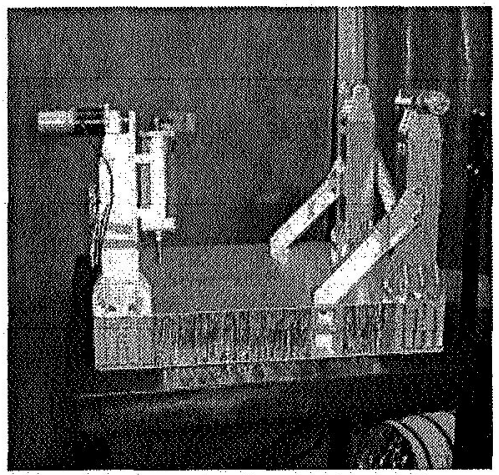

Figure 14, Qualification Unit Deployer Structure Alone, Prior to Integration on Pegasus Support Structure

The qualification unit alone is shown in Figure 14. The Pinpuller and pusher devices are seen on the left hand post, and the two hinges are on the right hand posts. 


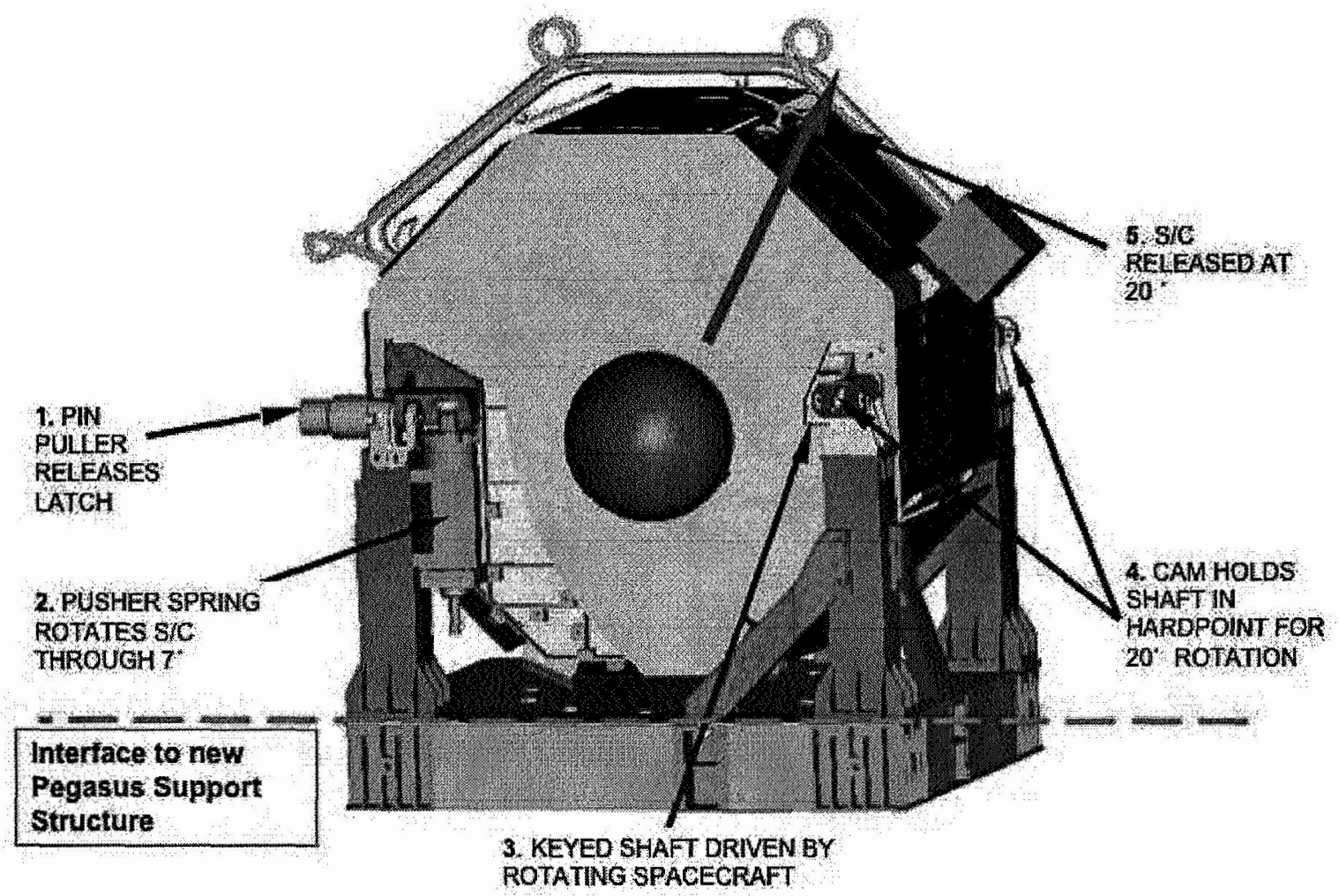

Figure 15, ST5 Spacecraft Deployer Structure Operation

\section{Deployer Structure Validation-}

The Deployer Structure design was validated for performance and environment both at the discreet qualification unit level, as shown in Figures $13 \& 14$, and at the integrated Pegasus Support Structure level, and shown previously and in Figure 15. Essentially, performance validation required that the device provide sufficient spin rate for the entire mission, including de-spin effects caused by the deploying boom, while environmental validation required sustaining the dynamic and thermal environments. Proof of this validation was supplied by analysis, inspection and test.

Key success criteria included

- The maximum nutation angle induced upon the spacecraft at deployment less than 10 degrees

- Verifying clean separation of the SC from the Deployer Structure. The hinges retracted before the SC completely cleared the posts, creating the possibility of contact. Such an event could damage a solar panel or perturb the spin or nutation angle.

- Assessing the translational velocities of the $\mathrm{SC}$ as they came out of the Deployer. Flight Dynamics predictions showed a slight possibility that the three SC could re-contact at a future point in the mission. Adequate control of the relative velocities was required to reduce this chance.
- Spinning the Spacecraft at $20 \pm 2 \mathrm{rpm}$ for the forward and aft SC, and middle SC spinning at 27 $\pm 2.7 \mathrm{RPM}$. These rates were backed out of the Flight Dynamics requirement for SC-to-SC separation to prevent re-contact in orbit.

- Deploying the SC under extremes of temperature. Since the release mechanism design was not fully kinematic due to the spin-and-release requirement, any temperature gradients within the structure could cause binding and failure to operate.

Using motion analysis software that duplicated the kinematics of the release mechanism and the free-flying SC, the clearance was found to be positive even with conservative, one-sided frictional interaction was considered. During ground test, high rate digital video imaging of the deployment (Figure 15) verified the analytical results. Gravity effects were negated with the counterweight system. A cable and counterweight system to simulate zero gravity conditions was utilized in deployments with the flight spacecraft. The deploy test was performed again after vibration to verify performance after environmental exposure. 


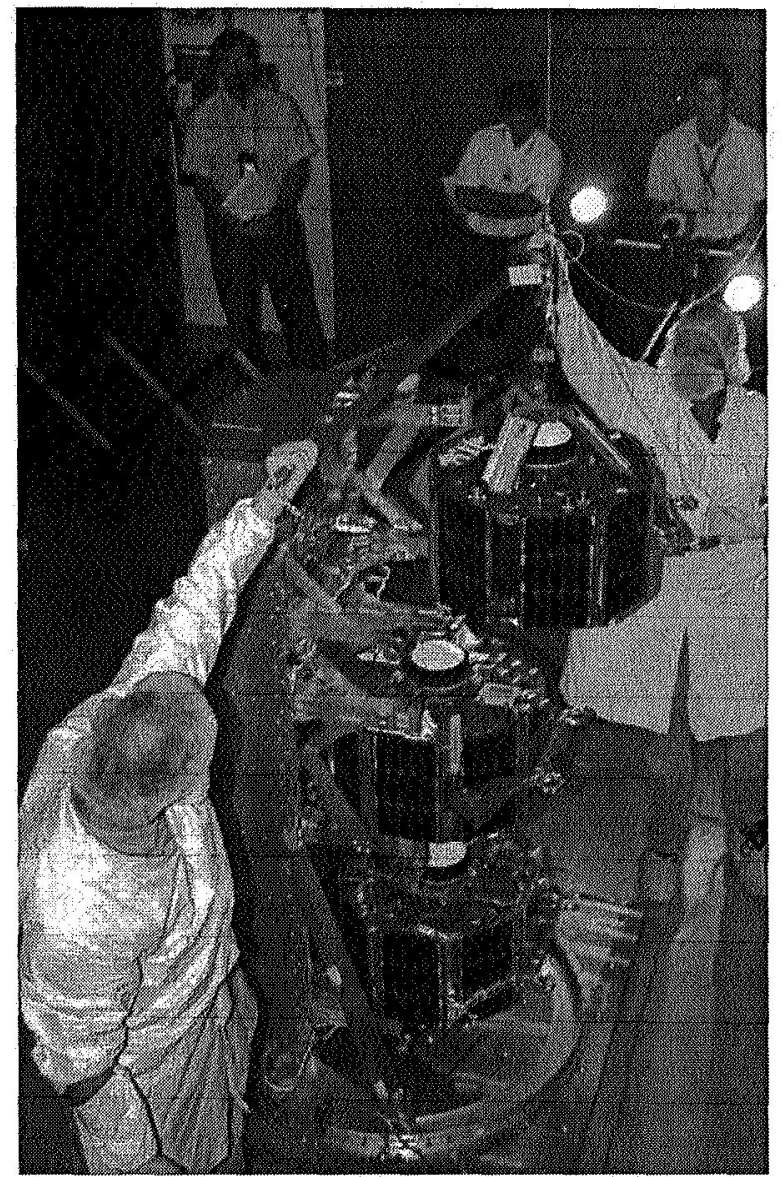

Figure 16, Test deployments of Flight SC in complete flight configuration in ambient

Both the qualification unit and the ProtoFlight PSS were vibration and thermal-vacuum tested. In the Thermal chamber (Figure 17), conservative gradients were applied to the mechanism base and the device was commanded to separate a SC simulator in order to verify proper operation. This occurred with a high-fidelity spacecraft mock-up, flight-like harnessing and blankets installed. In this test, Gnegation was provided by bottom mounted casters rolling on a flat plate.

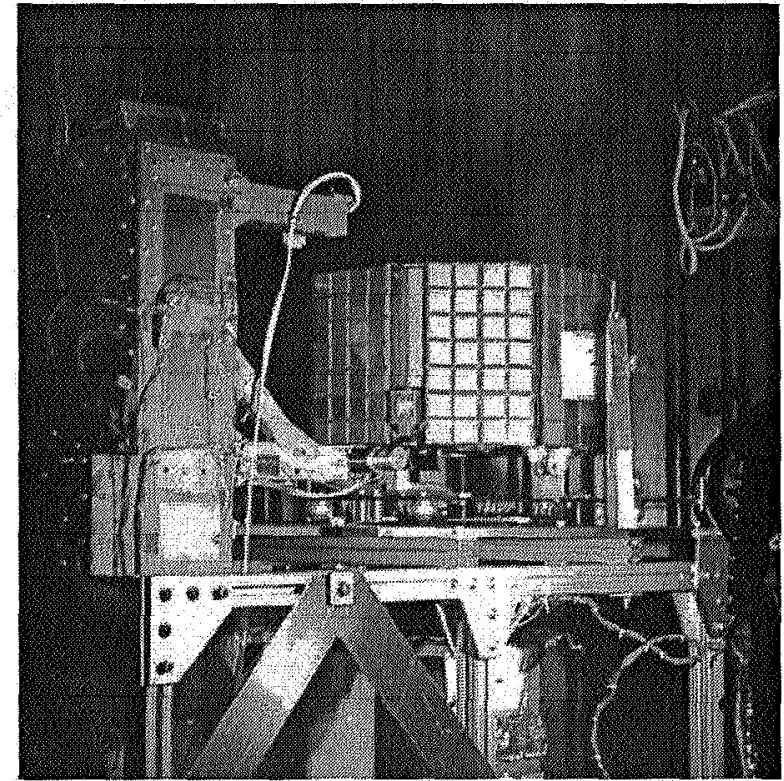

Figure 17, Thermal-vacuum and Temperature Gradient testing of the Flight PSS and SC simulator 


\section{ON-Orbit Performance}

\section{Day of launch}

At 6:22AM on March 22, 2006 the ST5 payload was dropped from the Pegasus Carrier Aircraft. Stage 1 ignition occurred 5 seconds later.

The spacecraft deployment started with the Forward spacecraft at 587 seconds after drop. Launch vehicle telemetry showed that the pusher caused a 5 degree body recoil before the Reaction Control system compensated and righted the system attitude in space. Since the spacecraft cleared the Deployer Structure posts before 1 degree of rotation (in less than 0.2 second), there was sufficient clearance to avoid contact.
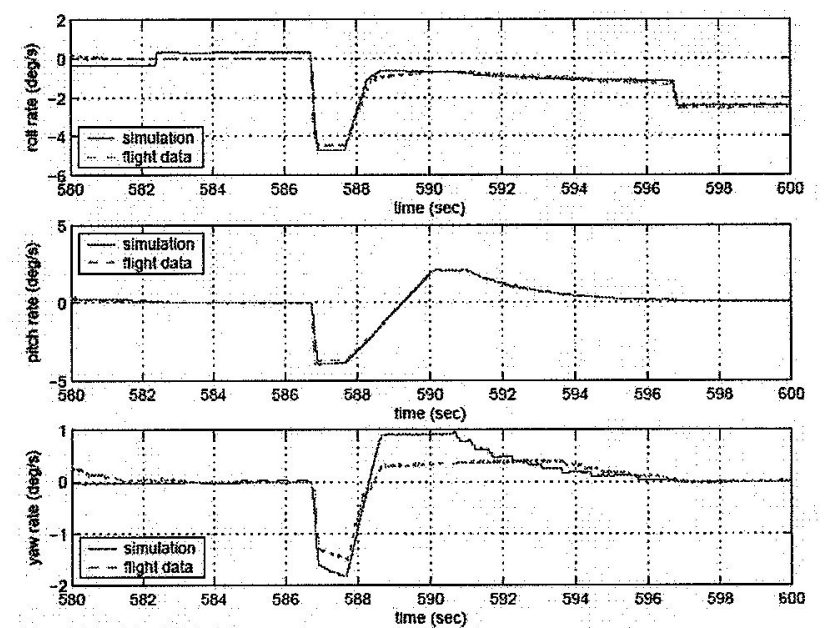

Figure 18 ST5 Forward Spacecraft Separation Body Rates

After deployment on orbit, the spacecraft's sun sensor measured coning angles shown in Table 1. The initial nutation angle refers to the spacecraft attitude immediately after separation from the deployer release mechanism, with tip-off, rigid-body interaction effects figuring into the equation.

Table 3 ST5 On-Orbit Spin Performance

\begin{tabular}{|c|c|c|c|c|}
\hline \multirow{2}{*}{$\begin{array}{c}\text { Spacecraf } \\
\mathbf{t}\end{array}$} & \multicolumn{2}{|c|}{ Spin Rate (rpm) } & \multicolumn{2}{|c|}{$\begin{array}{c}\text { Nutation Angles } \\
\text { (deg) }\end{array}$} \\
\cline { 2 - 5 } & Initial & Mission & Initial & Mission \\
\hline SC155 & 24.6 & 19.01 & 1.4 & $<1$ \\
\hline SC094 & 35.0 & 27.39 & 2.0 & $<1$ \\
\hline SC224 & 23.8 & 18.60 & 1.0 & $<1$ \\
\hline
\end{tabular}

\section{Mission Operations}

Initial Spin Rates -As the magnetometer booms were deployed the spacecraft spin-rate decreased. Mission spin rate is shown in Table 3 immediately after boom deployment. Pre-launch predictions of the final spacecraft spin-rates were very close, within $10 \%$, of the actual, final spin-rate, as measured by guidance, navigation and control telemetry.

The magnetometer boom deployed nominally, based on spacecraft telemetry, and the magnetometer data appears to be very stable. There have been no indications of fluctuations in the magnetometer's position with respect to the spacecraft.

Spin Rate Decay - Over the course of the mission, each SC spin rate was observed to decay. This was attributed to either atmospheric drag on the magnetometer boom near perigee or to eddy currents induced in the aluminum structure interacting with the Earth's magnetic field. The predominant mechanism for this phenomenon was proved to be magnetic interactions by test. The SC was commanded to rotate 90 degrees with respect to the magnetic field only, and the rate decay was found to decrease. The attitude thruster was co-aligned with the spin axis, so it had negligible effect on the spin rate.

\section{ConClusion}

The ST5 mechanical system functioned perfectly over the course of the mission. The six low-shock Pinpullers functioned as designed, releasing both a SC constellation and the Magnetometer Boom on command. The mechanical requirements of small-volume, low magnetic signature and spin balance were met.

The success of the mechanical system enabled the mission to demonstrate miniaturization of key satellite components such as thruster, X-Band communications, Ultra-Low Power CMOS circuitry and research-grade magnetometer science instrument. The science instrument confirmed a hypothesized low-frequency undulation in the Earth's magnetic field at low latitudes.

The mission was terminated on June 30, 2006 after 101 days. A Technology Validation Symposium was held at Goddard Space Flight Center on September 13, 2006 for stakeholders in the micro-satellite field [2]. This symposium summarized significant achievements and technology validation efforts. Interested parties may contact the author of further information. 


\section{REFERENCES}

[1] "Mission Requirements Document for ST5 Project," ST5-495-051 ${ }^{2}$

[2] STS Technology Validation Symposium presentation materials, September $13,2006^{2}$

\section{BIOGRAPHY}

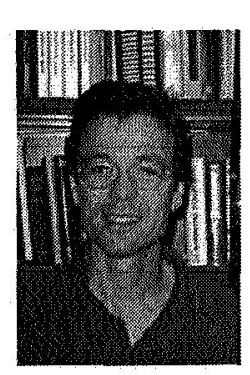

Peter Rossoni served as the Lead Engineer for the Space Technology 5 Mechanical System from the project proposal stage in 1999 to its launch in 2006. Just prior to joining the ST5 project, he performed and directed nano-satellite formulation efforts, including mission design for a 100 satellite constellation, nano-satellite bus concepts, and system conceptual studies. He has been directly supporting NASA missions as a design, structural dynamics and systems specialist for most of his NASA career. 